\title{
Pop III GRBs: an estimative of the event rate for future surveys
}

\section{Rafael S. de Souza*}

Korea Astronomy \& Space Science Institute, Daejeon 305-348, Korea Max-Planck-Institut für Astrophysik, Karl-Schwarzschild-Str. 1, D-85748 Garching, Germany E-mail: rafael@kasi.re.kr

\begin{abstract}
We discuss the theoretical event rate of gamma-ray bursts (GRBs) from the collapse of massive primordial stars. We construct a theoretical model to calculate the rate and detectability of these GRBs taking into account all important feedback and recent results from numerical simulations of pristine gas. We expect to observe a maximum of $\mathrm{N} \lesssim 0.2 \mathrm{GRBs}$ per year integrated over at $\mathrm{z}>6$ with Swift and $\mathrm{N} \lesssim 10$ GRBs per year integrated over at $\mathrm{z}>6$ with EXIST (assuming a sensitivity up to $10 \times$ higher than Swift).
\end{abstract}

Gamma-Ray Bursts 2012 Conference-GRB2012,

May 07-11, 2012

Munich, Germany

\footnotetext{
*Speaker.
} 


\section{Introduction}

The first stars (Pop III) in the Universe are likely to have played a major role in the early cosmic evolution, by emitting the first light and producing the first heavy elements [1,2]. It is of great importance to understand the origin and evolution of such objects, since their detection would permit to probe the pristine regions of the Universe.

Pop III stars may produce collapsar gamma-ray bursts (GRBs) whose total isotropic energy could be $\approx 2$ orders of magnitude larger than average [3, 4, 5]. Even if a Pop III star has a supergiant hydrogen envelope, the GRB jet can break out of it due to the long-lasting accretion of the envelope itself $[6,7]$.

\section{GRB redshift distribution}

The following is a brief overview of the model we used. Readers are encouraged to check [8] for a full description. As long GRBs are expected to follow the death of very massive stars, their rate provide an useful probe for cosmic star formation history $[8,9,10,11]$.

Over a particular time interval, $\Delta t_{\mathrm{obs}}$, in the observer rest frame, the number of observed GRBs originating between redshifts $z$ and $z+d z$ is

$$
\frac{\mathrm{d} N_{\mathrm{GRB}}^{\text {obs }}}{\mathrm{d} z}=\frac{\Omega_{\text {obs }}}{4 \pi} \eta_{\text {beam }} \eta_{\mathrm{GRB}} \Psi(z) \frac{\Delta t_{\mathrm{obs}}}{1+z} \frac{\mathrm{d} V}{\mathrm{~d} z} \int_{\log L_{\text {lim }}(z)}^{\infty} p(L) d \log L,
$$

where $\eta_{\mathrm{GRB}}$ is the GRB formation efficiency, $\Psi$ is the cosmic star formation rate (SFR) density, $\mathrm{d} V / \mathrm{d} z$ is the comoving volume element per redshift unit, $p(L)$ is the luminosity function, $\eta_{\text {beam }}$ is the beaming factor of the burst $\left(\sim 6^{\circ}\right)$ and $\Omega_{o b s}$ is the field of view of the experiment. The adopted values of $\Omega_{o b s}$ are 1.4, 2, 4, and 5 for Swift, SVOM, JANUS, and EXIST, respectively. The intrinsic GRB rate (the total number of GRBs per year on the sky ) is defined as $\frac{\mathrm{d} N_{\mathrm{GRB}}}{\mathrm{d} z}=\eta_{\mathrm{GRB}} \Psi(z) \frac{\Delta t_{\mathrm{obs}}}{1+z} \frac{\mathrm{d} V}{\mathrm{~d} z}$.

\subsection{Star Formation History}

In order to determine the star formation rate (SFR) at early epochs, we assume that stars are formed in collapsed dark matter halos. In what follows, we adopt the Sheth-Tormen mass function to estimate the number of dark matter halos, $n_{S T}(M, z)$, with mass less than $\mathrm{M}$ per comoving volume at a given redshift. The collapsed fraction of mass, $F_{\text {col }}(z)$, available for Pop III star formation is given by

$$
F_{\mathrm{col}}=\frac{1}{\rho_{m}} \int_{M_{H_{2}}}^{\infty} d M M n_{S T}(M, z) .
$$

Where $\rho_{m}$ is the total mass density of the background Universe. The star formation efficiency in the early Universe largely depends on the ability of a primordial gas to cool and condense. Hydrogen molecules $\left(\mathrm{H}_{2}\right)$ are the primary coolant in a gas in small mass "minihalos", and can be dissociated by soft ultraviolet radiation. Thus, a ultraviolet background in the Lyman-Werner (LW) bands can easily suppress the star formation inside mini-halos. We model the dissociation effect by setting the lower limit of equation (2.2) as the minimum halo mass capable of cooling by molecular hydrogen in the presence of a Lyman-Werner (LW) background (see [8] for more details). 
The star formation density can be described by the following expression,

$$
\Psi(z)=Q_{\mathrm{HII}} f_{\zeta} f_{*} \frac{\Omega_{b}}{\Omega_{m}} \frac{\mathrm{dF}_{\mathrm{col}}}{d t},
$$

where $\Omega_{\mathrm{m}}$ and $\Omega_{b}$ are the matter and baryon cosmological density parameters respectively. The factors $f_{\zeta}$ and $\mathrm{Q}_{\mathrm{HII}}$ represent the global filling fraction of metals via galactic winds and the volume filling fraction of ionized regions as shortly described below. The star formation efficiency ranges from $f_{*}=0.001$ as a conservative choice [12] to $f_{*}=0.1$ [13] as an upper limit. Given the large uncertainties on the above quantities we derive upper limits on the intrinsic GRB rate using $\sim 1$ year timescale radio variability surveys, which sets upper limits on the number of transients sources in the sky. Such limits can be used to ruled out our optimistic case. This is done by relating the intrinsic GRBs rate derived by our model with the number of afterglows in the sky, which is limited by observational limits from radio surveys (we are not really discussing how the constraints are derived here due to space limitation, please see [8] for details). Reionization: Inside growing HII regions, the gas is highly ionized and the temperature is $\sim 10^{4} \mathrm{~K}$. The volume filling factor of ionized regions, $Q_{\mathrm{HII}}(z)$, influences the typical mass of collapsed stars. Metal Enrichment $:$ The metal-enrichment in the inter-galactic medium (IGM) determines when the formation of primordial stars is terminated (locally) and switches from the Pop III mode to a more conventional mode of star formation. We follow the metal-enriched wind propagation outward from a central galaxy in order to evaluate the average metallicity over cosmic scales as a function of redshift. The top panel of Fig. 1 shows the upper limit for Pop III SFR adopted here.

\subsection{Initial Mass Function and GRB Formation Efficiency}

The stellar initial mass function (IMF) is critically important to determine the Pop III GRB rate. The IMF determines the fraction of stars with minimum mass which is able to trigger GRBs. The GRB formation efficiency factor per stellar mass can be written as

$$
\eta_{\mathrm{GRB}}=f_{\mathrm{GRB}} \int_{M_{\mathrm{GRB}}}^{M_{\mathrm{up}}} \phi(m) d m / \int_{M_{\mathrm{low}}}^{M_{\mathrm{up}}} m \phi(m) d m,
$$

The $f_{\mathrm{GRB}}$ factor gives the fraction of stars in this range of mass that will produce GRBs. And $\phi(m)$ is the stellar IMF which, we considered a Gaussian. We assume $\bar{M}=40 M_{\odot}$ and dispersion $\sigma_{c}=\left(\bar{M}-M_{\text {low }}\right) / 3 . M_{\text {low }}$ and $M_{\text {up }}$ are the minimum and maximum mass adopted for Pop III stars. Which ranges from $10 M_{\odot}$ to $100 M_{\odot}$ respectively. $M_{\mathrm{GRB}}$ is the minimum mass necessary to trigger GRBs, which we set to be $25 M_{\odot}[13]$.

\subsection{Luminosity function}

The number of GRBs detectable by any given instrument depends on the instrument-specific flux sensitivity threshold and also on the intrinsic isotropic luminosity function of GRBs. We adopt a power-law distribution function similar to [14]

$$
p(L)=\left(\frac{L}{L_{*}}\right)^{\alpha} L<L_{*},\left(\frac{L}{L_{*}}\right)^{\beta} L>L_{*},
$$




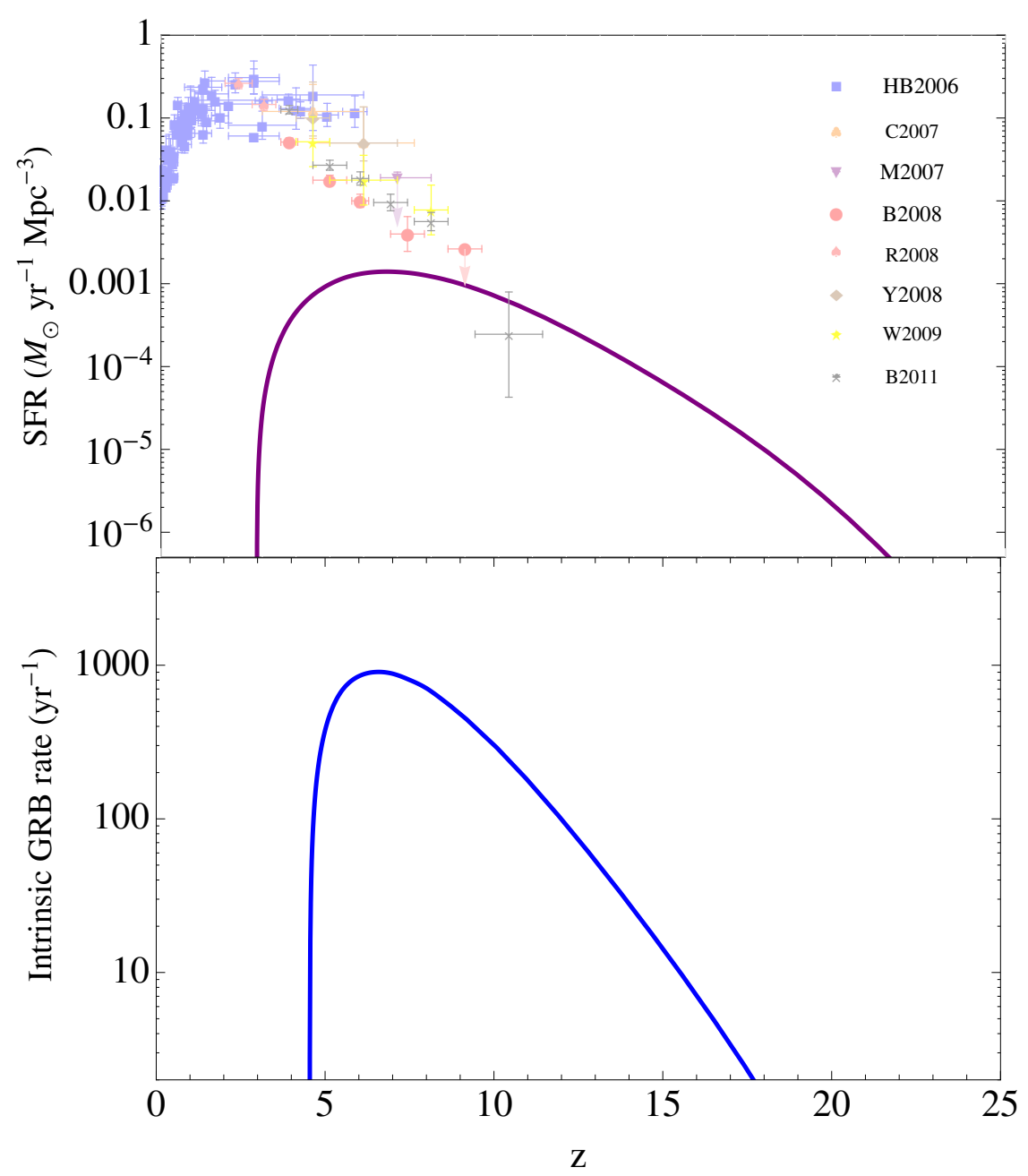

Figure 1: Top: Optimistic model for Pop III star formation rate. The light points are independent SFR determinations compiled from the literature.

Bottom: The intrinsic GRB rate $\mathrm{d} N_{\mathrm{GRB}} / \mathrm{d} z$. In other words, the number of GRBs per year on the sky (on-axis + off-axis).

where $L_{*}$ is the characteristic isotropic luminosity, $\alpha=-0.2_{-0.1}^{+0.2}$ and $\beta=-1.4_{-0.6}^{+0.3}$. The Pop III GRBs are assumed to be energetic with isotropic kinetic energy $E_{i s o} \sim 10^{56-57} \mathrm{erg}$ but long-lived, $T_{90} \sim 1000 \mathrm{~s}$. Thus the luminosity would be moderate, $L_{*} \sim \varepsilon_{\gamma} \times 10^{56-57} / 1000 \sim 10^{52-53} \mathrm{ergs} / \mathrm{s}$ if $\varepsilon_{\gamma} \sim 0.1$ is the conversion efficiency from the jet kinetic energy to gamma rays [6].

Using equation (2.5) we can predict the observable GRB rate for the Swift, SVOM, JANUS, and EXIST missions. For Swift, we set a bolometric energy flux limit $F_{\lim }=1.2 \times 10^{-8} \mathrm{erg} \mathrm{cm}^{-2} \mathrm{~s}^{-1}$. We adopt a similar limit for SVOM. For JANUS, $F_{\text {lim }} \sim 10^{-8} \mathrm{erg} \mathrm{cm}^{-2} \mathrm{~s}^{-1}$. The luminosity threshold is then $L_{\mathrm{lim}}=4 \pi d_{\mathrm{L}}^{2} F_{\text {lim. }}$. Here $d_{L}$ is the luminosity distance for the adopted $\Lambda$ CDM cosmology. Given that EXIST is expected to be $\sim 7-10$ times more sensitive than Swift, we set its sensitivity threshold ten times lower than Swift's as an approximate estimative. For simplicity, we assume that the spectral energy distribution (SED) peaks at X-to- $\gamma$ ray energy (detector bandwidth) as an optimistic case. We expect to observe a maximum of $\mathrm{N} \lesssim 10$ GRBs per year integrated over at $\mathrm{z}>$ 


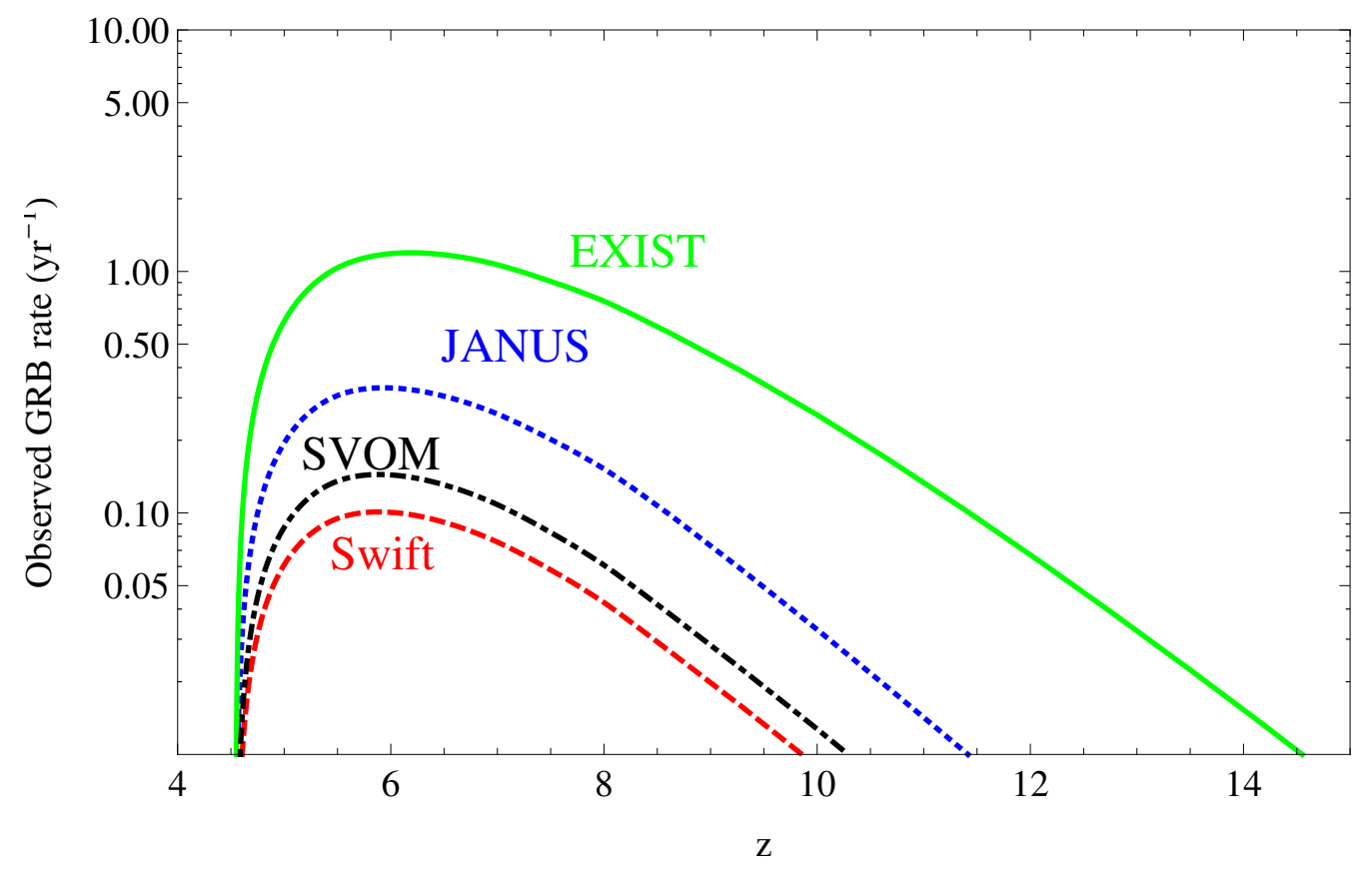

Figure 2: Predicted Pop III observed GRB rate, $\mathrm{d} N_{\mathrm{GRB}}^{o b s} / \mathrm{d} z$. Those observed by Swift, dashed red line; SVOM, dot-dashed black line; JANUS, dotted blue line; and EXIST, green line; for our model with Gaussian $\mathrm{IMF}, f_{*}=0.03, f_{G R B}=0.001$.

6 with EXIST, and $\mathrm{N} \lesssim 0.2$ GRBs per year integrated over at $\mathrm{z}>6$ with Swift.

\section{Summary}

GRBs can be observed at large distances, been a powerful tool to probe the early Universe in situ. Together with pair instability supernovae, they are probably the most promising way to observe the first stars. Here we discuss the observed rate of such objects in current and future surveys. We take into account the recent developments concerning the origin of Pop III stars, in particular the last results from [15], which show Pop III stars can be less massive than previously thought. We expect to observe a maximum of $\mathrm{N} \lesssim 10$ GRBs per year integrated over at $\mathrm{z}>6$ with EXIST, and $\mathrm{N} \lesssim 0.2$ GRBs per year integrated over at $\mathrm{z}>6$ with Swift. If we consider that we have no observation so far from Pop III GRBs among all GRBs observed by Swift with redshift measurements. We can place an upper limit over the fraction of Pop III GRBs over Pop II/I ones, $<1 / N_{\text {Swift }}$, where $N_{\text {Swift }}$ is the number of long GRBs with redshift measurements $(\sim 150)$. Which gives an fraction of $\sim 0.0067$. Thus, considering an average of 150/6 GRBs $y r s^{-1}(25$ GRBs $y r s^{-1}$ ) with redshift measurements. It gives un upper limit of $N \lesssim 0.56$ Pop III GRBs $y r^{-1}$ $(0.0067 \times 25 \times 1 / 0.3)$, taking into account that we only measure redshifts for $\sim 0.3$ of all GRBs. Which is consistent with our estimative.

It's important to note that even if such objects are above the flux limit threshold, most of the high-z GRBs might be hidden by dust and be "dark grbs". An alternative is to detect their afterglows. As their afterglows are expected to be long lasting $\sim 1000$ days, will probably be difficult to be detected them as transients. To overcome this problem, we should rely in surveys 
strategies that take snapshots from the sky during long and spaced periods of time. Several tools for photometric supernova classification already exist and have been tested with synthetic supernovae light curves [16]. We suggest that the same approach could be done in order to look for Pop III GRBs candidates in radio surveys.

\section{References}

[1] N. Yoshida, K. Omukai, L. Hernquist, Protostar Formation in the Early Universe, Science 321 (2008) 669

[2] V. Bromm, N. Yoshida, The First Galaxies, ARAA 49 (2011) 373

[3] S. S. Komissarov, M. V. Barkov, Supercollapsars and their X-ray bursts, MNRAS 402 (2010) L25.

[4] P. Mészáros, M. J. Rees, Population III Gamma-ray Bursts, ApJ 715 (2010) 967.

[5] K. Toma, T. Sakamoto, P. Mészáros, Population III Gamma-ray Burst Afterglows: Constraints on Stellar Masses and External Medium Densities , ApJ 731 (2011) 127.

[6] Y. Suwa, K. Ioka, Can Gamma-ray Burst Jets Break Out the First Stars?, ApJ 726 (2011) 107.

[7] H. Nagakura, Y. Suwa, K. Ioka, Population III Gamma-Ray Bursts and Breakout Criteria for Accretion-Powered Jets, ApJ 754 (2012) 85.

[8] R. S. de Souza, N. Yoshida, K. Ioka, Populations III.1 and III.2 gamma-ray bursts: constraints on the event rate for future radio and $X$-ray surveys, $A \& A, \mathbf{5 3 3}$ (2011) A32.

[9] E. E. O. Ishida, R. S. de Souza, A. Ferrara, Probing cosmic star formation up to $z=9.4$ with gamma-ray bursts, MNRAS $\mathbf{4 1 8}$ (2011) 500

[10] R. S. de Souza, A. Krone-Martins, E. E. O. Ishida, B. Ciardi, Searching for the first stars with the Gaia mission, (2011) [astro-ph/1112.6270]

[11] B. E. Robertson, R. S. Ellis, Connecting the Gamma Ray Burst Rate and the Cosmic Star Formation History: Implications for Reionization and Galaxy Evolution, ApJ 744 (2012) 95.

[12] T. H Greif, V. Bromm, Two populations of metal-free stars in the early Universe MNRAS 373 (2006) 128.

[13] V. Bromm, A. Loeb High-Redshift Gamma-Ray Bursts from Population III Progenitors, ApJ 642 (2006) 382.

[14] D. Wanderman, T. Piran, The luminosity function and the rate of Swift's gamma-ray bursts, MNRAS 406 (2010) 1944.

[15] T. Hosokawa, K. Omukai, N. Yoshida, H. W., Yorke Protostellar Feedback Halts the Growth of the First Stars in the Universe, Science 334 (2011) 1250.

[16] E. E. O. Ishida, R. S. de Souza, Kernel PCA for type Ia supernovae photometric classification, (2012) [astro-ph/1201.6676] 